\title{
Infrared-assisted extraction followed by high performance liquid chromatography to determine angoroside C, cinnamic acid, and harpagoside content in Scrophularia ningpoensis
}

\author{
Lina Su', Yinghui Deng ${ }^{1}$, Nianzu Chen ${ }^{2}$, Xiuwen Zhang ${ }^{2}$ and Taomin Huang ${ }^{2 *}$ (D)
}

\begin{abstract}
Background: Angoroside C, cinnamic acid, and harpagoside are bioactive constituents in Scrophularia ningpoensis. Currently, an infrared-assisted extraction (IRAE) method coupled with high-performance liquid chromatography with ultraviolet detection (HPLC-UV) for the analysis of bioactive constituents in this plant is lacking.

Methods: A method based on HPLC following IRAE has been developed for quantifying angoroside C, cinnamic acid, and harpagoside in Scrophularia ningpoensis. Four main factors, namely, extraction solvent, solid/liquid ratio, illumination time, and distance between the infrared lamp and the round-bottom flask, were optimized for extraction. Furthermore, conventional ultrasonic extraction (USE) and microwave-assisted extraction (MAE) were also investigated to validate the developed method.

Results: The optimal extraction conditions were as follows: ethanol concentration, 37.5\%; solid/liquid ratio, 1:25; illumination time, $10 \mathrm{~min}$; and distance between infrared lamp and round-bottom flask, $3 \mathrm{~cm}$. The results of method validation demonstrated that the developed method meets the requirement of analysis.
\end{abstract}

Conclusion: The results show that the IRAE-HPLC is a simple, accurate, and green analytical preparatory method for the potential extraction and quantification of angoroside C, cinnamic acid, and harpagoside in Scrophularia ningpoensis.

Keywords: Angoroside C, Cinnamic acid, Harpagoside, Infrared-assisted extraction (IRAE), Scrophularia ningpoensis, Traditional Chinese medicine

\section{Background}

Scrophularia ningpoensis Hemsl (Xuanshen in Chinese, S. ningpoensis), a traditional Chinese medicine (TCM) plant, recorded in the Compendium of Materia Medica and Pharmacopoeia of China [1], has been prescribed to treat various diseases for thousands of years. In clinical practice, it is commonly used to treat pharyngalgia, rheumatism, arthritis, tussis, constipation, and conjunctival congestion [1-3]. It is especially effective for the

\footnotetext{
* Correspondence: taominhuang@126.com

${ }^{2}$ Department of Pharmacy, Eye \& ENT Hospital, Fudan University, Shanghai 200031, China

Full list of author information is available at the end of the article
}

throat and vocal cord. To ensure the safety and efficiency of S. ningpoensis, quality control is critical [4].

Angoroside C, cinnamic acid, and harpagoside (Fig. 1) are the main bioactive components in S. ningpoensis. Angoroside $\mathrm{C}$ has anti-inflammatory, anti-oxidation, platelet-aggregation inhibition, and liver protection effects [5]. Harpagoside has hypotensive, anti-hepatitis B virus (HBV), anti-inflammatory, anti-arrhythmic, and positive inotropic effects [6-10]. While cinnamic acid has been shown to possess anti-fungal [11] and antioxidant activities [12], as well as the ability to induce tumor cell differentiation [13].

Given that angoroside C, cinnamic acid, and harpagoside are the key bioactive components of TCMs, it is 


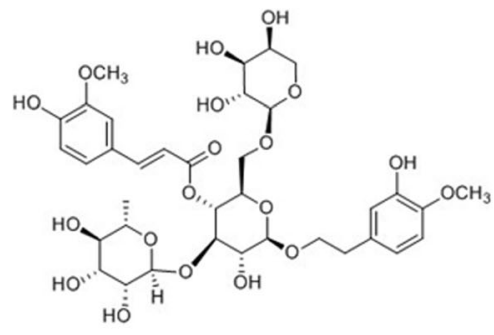

a

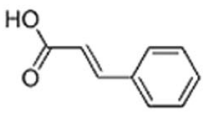

b

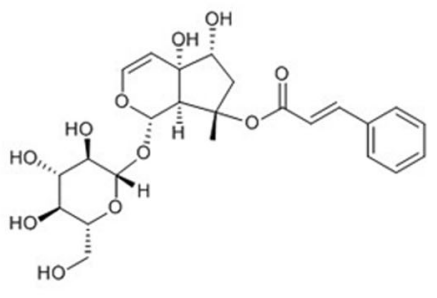

Fig. 1 Chemical structures of (a) angoroside C, (b) cinnamic acid and (c) harpagoside

essential to determine angoroside $\mathrm{C}$, cinnamic acid, and harpagoside abundance in TCMs. This requires the development of a simple, accurate analytical method for quantifying angoroside $\mathrm{C}$, cinnamic acid, and harpagoside in S. ningpoensis.

Several methods, such as high performance liquid chromatography (HPLC) $[1,10,14-19]$ and capillary electrophoresis [20] have been developed for quantifying angoroside $\mathrm{C}$, cinnamic acid, and harpagoside in plants, medicinal preparations, and biological samples. Prior to quantification, it is necessary to isolate and extract angoroside $\mathrm{C}$, cinnamic acid, and harpagoside from TCMs. Various extraction techniques have been proposed, including Soxhlet extraction, steam distillation, hydro-distillation, and solvent extraction [21-25]. However, these extraction methods have limitations, such as low extraction efficiency and toxic solvent residue in the extract. Moreover, these extraction procedures are timeconsuming. New techniques exist, such as supercritical fluids, microwave, and IRAE methods, which typically consume less solvent, time, and energy.

Infrared technology is ubiquitous in everyday life. Numerous consumer goods, including food, medical devices, television, and mobile phones rely on infrared technology. Infrared light, an electromagnetic wave, has the advantages of high permeability, low energy consumption, rapid heating, and safe operation. Furthermore, it has the advantage of high extraction efficiency of analytes compared with that of the conventional techniques [24, 26, 27]. Currently, high- performance liquid chromatography with ultraviolet detection (HPLC-UV), as an analytical method, is widely used. Thus, developing an IRAE method coupled with HPLC-UV for analyzing the bioactive constituents in S. ningpoensis is important.

In this study, IRAE followed by HPLC was developed for the quantitative analysis of angoroside C, cinnamic acid, and harpagoside in S. ningpoensis. The extraction conditions were optimized and the method was validated.

\section{Methods}

\section{Materials and reagents}

The dried roots of S. ningpoensis were purchased from the Anhui Dechang Pharmaceutical Co., Ltd. (Anhui, China) and ground to a fine powder. Angoroside $\mathrm{C}$ (analytical grade; lot no. 151205, purity $\geq 98 \%$ ) (Fig. 1a) was obtained from Shanghai Kangbiao Chemicals Co., Ltd. (Shanghai, China). Cinnamic acid (analytical grade; lot no. 111730200,604) (Fig. 1b) and harpagoside (analytical grade, lot no. 110786-200,503) (Fig. 1c) were purchased from the National Institute for the Control of Pharmaceuticals and Biological Products (Beijing, China). Acetic acid was obtained from Revitalization of Chinese Chemical Plant (Jiangsu, China). Methanol (HPLC grade) was purchased from Merck (New Jersey, USA). Deionized water was purified using an Auto ScienceAP-01P System from Tianjin Automatic Science Instrument Co., Ltd. (Shanghai, China). The infrared lamp (275 W) was obtained from Shanghai Tour Light Electrical Appliance Co. Ltd. (Shanghai, China).

\section{IRAE procedure}

The apparatus for IRAE, according to our previously reported method, is illustrated in Fig. 2 [28]. During the extraction process, the conduit for cooling water was

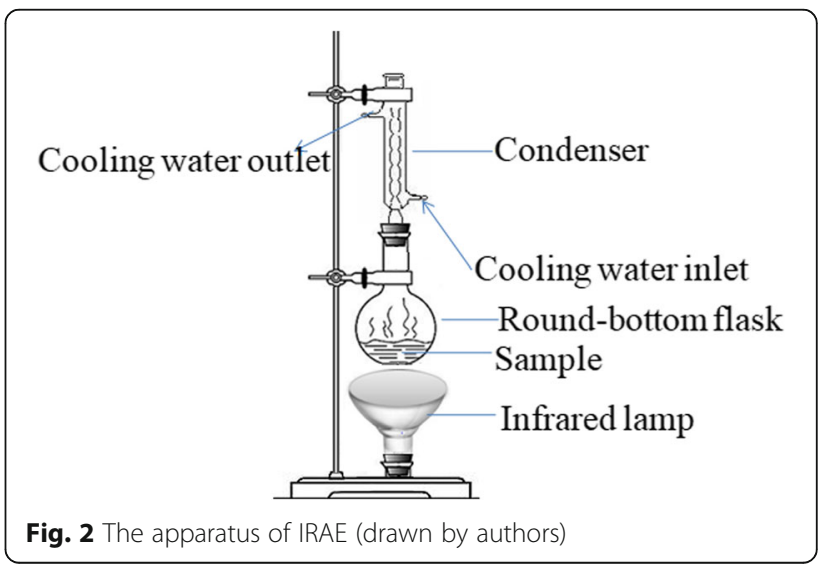


connected to the condenser to prevent solvent evaporation.

One gram of $S$. ningpoensis dried root was accurately weighed, transferred into a $100-\mathrm{mL}$ round-bottom flask containing $25 \mathrm{~mL}$ of $37.5 \%$ ethanol-distilled solvent, and extracted under illumination for $10 \mathrm{~min}$ with a distance of $3 \mathrm{~cm}$. The flask was accurately weighed before and after extraction.

\section{MAE procedure}

According to our previously reported method [1, 28], $1 \mathrm{~g}$ of $S$. ningpoensis dried root was placed in a $100-\mathrm{mL}$ flask with $25 \mathrm{~mL}$ of $37.5 \%$ ethanol-distilled solvent (the optimum extraction solvent obtained using the IRAE method). The flask with the sample was placed in an MO-2270 M1 model microwave oven and heated at 400 W for $4 \mathrm{~min}$. Simultaneously, a condenser with a continuous flow of cooling water was connected to condense the solvent vapor.

\section{USE procedure}

According to our previously reported method [28], $1 \mathrm{~g}$ of $S$. ningpoensis dried root was transferred into a 100$\mathrm{mL}$ flask containing $25 \mathrm{~mL}$ of $37.5 \%$ ethanol-distilled solvent (the optimum extraction solvent obtained using the IRAE method). The irradiation time was $60 \mathrm{~min}$.

\section{Calibration solution preparation}

Stock solutions $(1 \mathrm{mg} / \mathrm{mL})$ of angoroside $C$, cinnamic acid, and harpagoside were prepared by dissolving them in methanol. Working standard solutions of concentrations $5,10,25,50,100$, and $200 \mu \mathrm{g} / \mathrm{mL}$ for angoroside $\mathrm{C}$ and harpagoside, $2.5,5,12.5,25,50$, and $100 \mu \mathrm{g} / \mathrm{mL}$ for cinnamic acid were prepared by diluting the respective stock solutions with methanol:water (50:50). The samples were stored at $4{ }^{\circ} \mathrm{C}$. The calibration curves were obtained by weighted linear regression (weighing factor $1 / x)$; the peak area was plotted versus the analyte concentration.

\section{HPLC analysis}

An Agilent (Palo Alto, CA, USA) 1100 LC system, equipped with a G1311A Quatpump, a G1322A vacuum degasser, a G1316A Thermostatted Column Compartment, a G1314A variable wavelength UV-visible detector, and an HP 1100 series manual injector with a $20-\mu \mathrm{L}$ fixed loop, was used for the analysis. The detector was operated at $278 \mathrm{~nm}$ and peak areas were integrated automatically using Hewlett-Packard ChemStation software program (Rev. A. 10. 02 [1757]).

An Agilent TC C18 column $(200 \mathrm{~mm} \times 4.6 \mathrm{~mm}$ i.d., $5 \mu \mathrm{m}$ particle size) was used to separate the analytes. The mobile phase consisted of solvents A $(0.2 \%$ acetic acid) and $B$ (methanol). The gradient elution steps were from $65: 35$ to $40: 60$ (A:B) over 15 min with a flow rate of $1 \mathrm{~mL} / \mathrm{min}$ and column temperature of $25^{\circ} \mathrm{C}$. After each run, the column was re-equilibrated for $5 \mathrm{~min}$. Peak areas were used for quantification.

\section{Validation of the HPLC method}

To validate the method, the linearity, detection limit, repeatability, accuracy, and recovery were evaluated. The intra- and inter-day precision was determined by analyzing calibration samples during a single day and on three consecutive days, respectively. The relative standard deviation (RSD, \%) was calculated based on the obtained peak area.

Solutions prepared using $S$. ningpoensis sample $(n=6)$ were used to analyze the reproducibility of the method. The accuracy of this method was evaluated using a recovery test. Accurate amounts of the reference compounds were transferred to $S$. ningpoensis sample, and then extracted and analyzed using the developed method. The recovery was calculated using the following formula: recovery $(\%)=$ (amount found-original amount) / amount added $\times 100(n=6)$.

The limit of detection (LOD) and limit of quantification (LOQ) were determined by serially diluting the standard solution to different concentrations with methanol:water (50:50). The signal-to-noise $(\mathrm{S} / \mathrm{N})$ value of LOD and LOQ was 3 and 10, respectively. Ruggedness was examined using the same batch sample $(n=6)$. The stability of angoroside $C$, cinnamic acid, and harpagoside was investigated by periodic analysis of the same sample.

\section{Quantification of angoroside C, cinnamic acid, and harpagoside in $\mathrm{S}$. ningpoensis}

The sample was centrifuged at $1200 \times g$ for $10 \mathrm{~min}$. The supernatant was passed through a $0.45-\mu \mathrm{m}$ filter membrane. The sample was analyzed using an HPLC system. The results of IRAE were compared with those of the MAE and USE methods.

\section{Results}

Optimization of IRAE parameters

Effect of extraction solvent on the extraction efficiency of angoroside $C$, cinnamic acid, and harpagoside

Solutions containing different proportions $(0,12.5,25$, $37.5,50$, and $75 \%$ ) of ethanol were optimized under illumination for $10 \mathrm{~min}$ at a solid/liquid ratio of 1:25. The extraction efficiency of angoroside $C$, cinnamic acid, and harpagoside increased with increase in the ethanol ratio from 0 to $37.5 \%$, reaching a maximum at $37.5 \%$, and then decreasing from 37.5 to $75 \%$ (Fig. 3a). Therefore, $37.5 \%$ ethanol solution was selected as the extraction solvent of angoroside $\mathrm{C}$, cinnamic acid, and harpagoside in the subsequent experiment. 

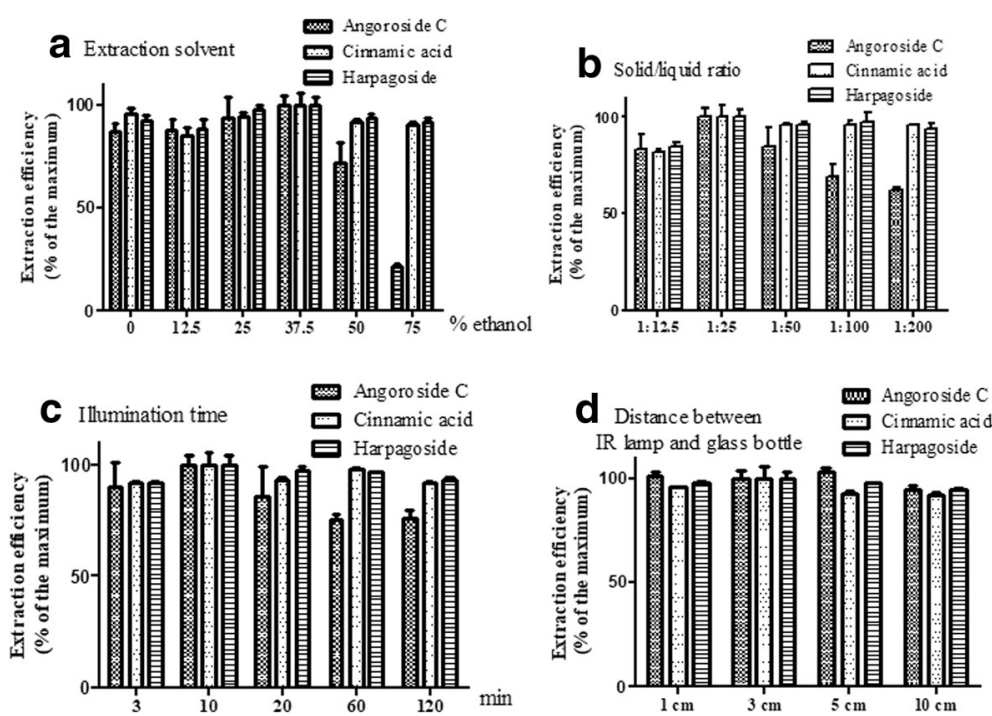

Fig. 3 Effects of the experiment parameters on extraction yield of angoroside C, cinnamic acid and harpagoside from S. ningpoensis in IRAE ( $n=3$ ). a Effects of solvent. Ratio of solid/liquid: 1:25; illumination time: $10 \mathrm{~min}$; the distance: $3 \mathrm{~cm}$. b Effects of solid/liquid ratio. Extraction solvent: $37.5 \%$ ethanol solution; illumination time: $10 \mathrm{~min}$; the distance: $3 \mathrm{~cm}$. c Effects of illumination time. Extraction solvent: $37.5 \%$ ethanol solution; Ratio of solid/liquid: 1:25; the distance: $3 \mathrm{~cm}$. $\mathbf{d}$ Effects of distance between the IR lamp and glass flask. Extraction solvent: $37.5 \%$ ethanol solution; Ratio of solid/liquid: 1:25; illumination time: $10 \mathrm{~min}$

\section{Effect of solid/liquid ratio on the extraction efficiency of angoroside $C$, cinnamic acid, and harpagoside}

The solid/liquid ratios of 1:12.5, 1:25, 1:50, 1:100, and 1: 200 (amount of material in g/volume of extraction solvent in $\mathrm{mL}$ ), were optimized with $37.5 \%$ ethanol under illumination for $10 \mathrm{~min}$. The extraction efficiency of angoroside $\mathrm{C}$, cinnamic acid, and harpagoside reached a maximum when the solid/liquid ratio was 1:25 (Fig. 3b). Therefore, the solid/liquid ratio of 1:25 was selected for IRAE in the subsequent analyses.

\section{Effect of illumination time on the extraction efficiency of angoroside $C$, cinnamic acid, and harpagoside}

The illumination time (3, 10, 20, 60, and $120 \mathrm{~min}$ ) was optimized with $37.5 \%$ ethanol solution, at a solid/liquid ratio of 1:25. The results showed that the extraction efficiency increased with the illumination time from 3 to $10 \mathrm{~min}$, and then decreased from 10 to $120 \mathrm{~min}$ (Fig. 3c). Hence, the best extraction efficiency of IRAE was obtained with illumination for $10 \mathrm{~min}$.

\section{Effect of distance between the IR lamp and the round- bottom flask}

The amount of extracted angoroside C, cinnamic acid, and harpagoside decreased with distance between the IR lamp and the round-bottom flask from 5 to $10 \mathrm{~cm}$. Although there were no significant differences between angoroside $\mathrm{C}$ and harpagoside at a distance of 3 and 5 $\mathrm{cm}$ in terms of yield, cinnamic acid had a better extraction yield at $3 \mathrm{~cm}$. A distance of $3 \mathrm{~cm}$ was chosen as the optimum distance (Fig. 3d).

\section{Method validation}

Figure 4 shows the representative chromatograms of the angoroside $\mathrm{C}$, cinnamic acid, and harpagoside (equivalent to $25 \mu \mathrm{g} / \mathrm{mL}$ ) standards, and $S$. ningpoensis sample. The resolution values, symmetry, and theoretical plates of angoroside $\mathrm{C}$, cinnamic acid, and harpagoside were above 3, 0.8, and 5000, respectively. The retention time of angoroside $\mathrm{C}$, cinnamic acid, and harpagoside was $10.7,13.5$, and $17.9 \mathrm{~min}$, respectively.

The linearity range of angoroside $\mathrm{C}$, cinnamic acid, and harpagoside was 5-200 $(5,10,25,50,100$, and $200 \mu \mathrm{g} / \mathrm{mL}), 2.5-100 \mu \mathrm{g} / \mathrm{mL}(2.5,5,12.5,25,50$, and $100 \mu \mathrm{g} / \mathrm{mL})$, and 5-200 $(5,10,25,50,100$, and $200 \mu \mathrm{g} /$ $\mathrm{mL})$, respectively.

The calibration curves (Additional file 1: Figure S1) of angoroside C, cinnamic acid, and harpagoside were as follows: $y=21.86 x-7.66(r=0.9998), y=152.60 x+10.41$ $(r=0.9998)$, and $y=44.99 x-11.97(r=0.9997)$, respectively ( $n=3$; $y$ : peak area; $x$ : concentration, $\mu \mathrm{g} / \mathrm{mL})$.

All the RSD values of intra- and inter-day precision (Additional file 2: Figure S2, Additional file 3: Figure S3, Additional file 4: Figure S4), reproducibility, and recovery (Additional file 5: Figure S5) were less than 3\% (Tables 1 and 2). The LOQ (Additional file 6: Figure S6) of angoroside $\mathrm{C}$, cinnamic acid, and harpagoside was $2.5,1$, and $1.5 \mu \mathrm{g} / \mathrm{mL}$, respectively $(S / N=10)$, which were considerably lower than the concentration in $S$. 

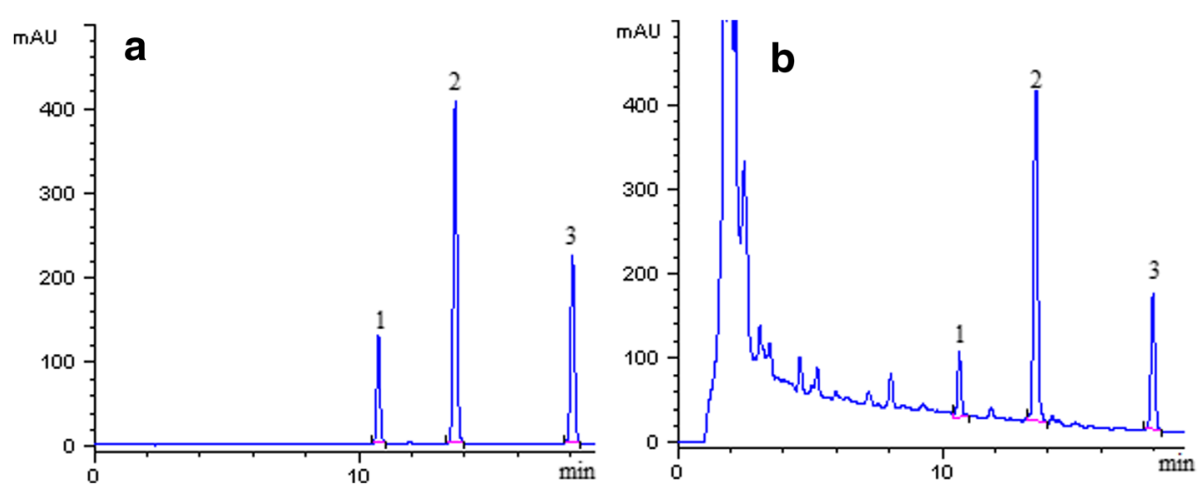

Fig. 4 Representative HPLC chromatogram of the standard solution at middle concentration (a) and real sample extracted from S. ningpoensis (b). $1=$ angoroside $C ; 2=$ cinnamic acid; $3=$ harpagoside

ningpoensis. The results demonstrated that the developed method is sensitive enough to analyze angoroside C, cinnamic acid, and harpagoside in S. ningpoensis.

The stability value of angoroside $\mathrm{C}$, cinnamic acid, and harpagoside was $1.29,1.67$, and $0.63 \%$, determined by periodic analysis of the same sample $(0,2,4,8$, and $24 \mathrm{~h})$, respectively.

\section{Determination of angoroside $\mathrm{C}$, cinnamic acid, and harpagoside in $\mathrm{S}$. ningpoensis sample}

Figure $4 \mathrm{~b}$ presents the HPLC chromatogram of angoroside $\mathrm{C}$, cinnamic acid, and harpagoside in S. ningpoensis sample obtained by IRAE under the optimal conditions. According to the calibration curves, the concentration of angoroside $\mathrm{C}$, cinnamic acid, and harpagoside in $S$. ningpoensis was calculated, and the analytical results of IREA, MAE, and USE are listed in Table 3. There was no significant difference in the respective concentrations

Table 1 Precisions of angoroside C, cinnamic acid and harpagoside

\begin{tabular}{lllll}
\hline $\begin{array}{llll}\text { Concentration } \\
\mu \mathrm{g} / \mathrm{mL})\end{array}$ & \multicolumn{2}{l}{ Intra-day $(n=3)$} & $\begin{array}{l}\text { Inter-day } \\
(n=3)\end{array}$ \\
\cline { 2 - 4 } Found & RSD $(\%)$ & Accuracy $(\%)$ & $\begin{array}{l}\text { RSD }(\%) \\
\text { Angoroside C }\end{array}$ \\
5 & $5.31 \pm 0.09$ & 1.72 & 106.16 & 1.88 \\
50 & $51.37 \pm 0.76$ & 1.50 & 102.74 & 1.69 \\
200 & $203.45 \pm 1.21$ & 0.60 & 101.72 & 0.63 \\
Cinnamic acid & & & & \\
2.5 & $2.58 \pm 0.04$ & 1.54 & 103.35 & 2.29 \\
25 & $26.07 \pm 0.11$ & 0.42 & 104.27 & 1.70 \\
100 & $100.32 \pm 0.65$ & 0.64 & 100.32 & 1.16 \\
Harpagoside & & & & \\
5 & $5.26 \pm 0.12$ & 2.31 & 105.25 & 2.06 \\
50 & $51.75 \pm 0.18$ & 0.35 & 103.50 & 1.46 \\
200 & $203.94 \pm 1.34$ & 0.66 & 101.97 & 0.99 \\
\hline
\end{tabular}

between the samples obtained using the IRAE and MAE methods. The concentration of angoroside $\mathrm{C}$, cinnamic acid, and harpagoside in S. ningpoensis sample obtained using the proposed method was significantly higher than the respective concentrations in the samples obtained using the USE method $(p<0.05)$.

\section{Discussion}

Scrophularia ningpoensis was extracted using the IRAE method, and then cooled and centrifuged at $1200 \times g$ for $10 \mathrm{~min}$, and analyzed by HPLC. The HPLC parameters were optimized by changing the components of the mobile phase. When the mixtures of methanol/acetonitrile and water were used as the mobile phase, angoroside $C$, cinnamic acid, and harpagoside were not well separated. When acetic acid was added into the mobile phase, the resolution value, symmetry, and theoretical plate of angoroside $\mathrm{C}$, cinnamic acid, and harpagoside were above $3,0.8$, and 5000 , respectively. The optimum mobile phase was achieved with an aqueous phase (containing $0.2 \%$ acetic acid).

The conventional MAE and USE methods were also investigated to demonstrate the reliability of the proposed method. The MAE parameters were also assessed. Scrophularia ningpoensis samples were extracted at different microwave powers $(200,400$, and $700 \mathrm{~W})$, different solid/liquid ratio (1:12.5, 1:25, 1:50, and 1:100), and under different irradiation times $(1,2,4$, and 6 $\mathrm{min}$ ) in $37.5 \%$ ethanol solution to determine the optimal conditions. It was found that the extraction efficiency of angoroside $\mathrm{C}$, cinnamic acid, and harpagoside reached the maximum at the solid/liquid ratio of 1:25, microwave power of $400 \mathrm{~W}$, and under irradiation for $4 \mathrm{~min}$.

Scrophularia ningpoensis samples were extracted at different solid/liquid ratio (1:12.5, 1:25, 1:50, and 1:100) under different irradiation times (30,60, and $90 \mathrm{~min}$ ) in $37.5 \%$ ethanol solution with an ultrasonic power of 
Table 2 Reproducibility and recovery of angoroside C, cinnamic acid and harpagoside

\begin{tabular}{llllll}
\hline Analyte & Original $(\mathrm{mg} / \mathrm{g})$ & Added $(\mathrm{mg} / \mathrm{g})$ & Recorded $(\mathrm{mg} / \mathrm{g})$ & Recovery $(\mathrm{mean}, \%)$ & RSD $(\%, n=6)$ \\
\hline Angoroside C & 0.83 & 1.25 & 1.30 & $104.47 \%$ & 0.64 \\
Cinnamic acid & 0.76 & 0.625 & 0.61 & $98.28 \%$ & 1.17 \\
Harpagoside & 0.94 & 1.25 & 1.31 & $104.55 \%$ & 0.58 \\
\hline
\end{tabular}

$90 \mathrm{~W}$. The optimum extraction condition by USE was as follows: solid/liquid ratio of 1:25 under irradiation for $60 \mathrm{~min}$.

Compared with that of the conventional USE method, IRAE provided a high extraction yield of angoroside $\mathrm{C}$, cinnamic acid, and harpagoside from $S$. ningpoensis sample. This result is in accordance with our previous study findings with IRAE [28]. Moreover, the IRAE method needs a shorter extraction time than that of USE and pollutes the environment less than that of USE.

Angoroside $\mathrm{C}$, cinnamic acid, and harpagoside are the main bioactive components in S. ningpoensis, and play an important role in treating diseases. To ensure the safety and efficacy, the quality of $S$. ningpoensis can be controlled by analyzing the concentrations of active constituents. Therefore, the proposed method has the potential for quality monitoring of TCMs in the future.

\section{Conclusions}

In the present study, the IRAE method coupled with HPLC has been developed to quantify angoroside C, cinnamic acid, and harpagoside in S. ningpoensis. The results of method validation demonstrated that the developed method meets the requirement of analysis. Because of the relatively lower detection limit, less extraction time was needed and higher extraction

Table 3 The concentrations of angoroside C, cinnamic acid and harpagoside in S. ningpoensis samples using different extraction methods under their optimal conditions ( $\mathrm{mg} / \mathrm{g} \pm \mathrm{SD}, n=3$ )

\begin{tabular}{llll}
\hline & \multicolumn{1}{l}{ IRAE } & MAE & USE \\
\hline Angoroside C $(\mathrm{mg} / \mathrm{g})$ & & \\
Batch 1 & $0.83 \pm 0.007$ & $0.84 \pm 0.024$ & $0.76 \pm 0.019$ \\
Batch 2 & $0.81 \pm 0.032$ & $0.84 \pm 0.009$ & $0.77 \pm 0.004$ \\
Batch 3 & $0.81 \pm 0.014$ & $0.75 \pm 0.018$ & $0.76 \pm 0.007$ \\
Cinnamic acid $(\mathrm{mg} / \mathrm{g})$ & & \\
Batch 1 & $0.76 \pm 0.019$ & $0.78 \pm 0.004$ & $0.71 \pm 0.009$ \\
Batch 2 & $0.77 \pm 0.004$ & $0.76 \pm 0.015$ & $0.71 \pm 0.001$ \\
Batch 3 & $0.76 \pm 0.007$ & $0.76 \pm 0.007$ & $0.71 \pm 0.005$ \\
Harpagoside $(\mathrm{mg} / \mathrm{g})$ & & \\
Batch 1 & $0.94 \pm 0.006$ & $0.94 \pm 0.002$ & $0.86 \pm 0.008$ \\
Batch 2 & $0.94 \pm 0.006$ & $0.93 \pm 0.007$ & $0.87 \pm 0.001$ \\
Batch 3 & $0.94 \pm 0.006$ & $0.95 \pm 0.002$ & $0.86 \pm 0.007$ \\
\hline
\end{tabular}

efficiency was achieved. The IRAE method proposed to quantify angoroside $\mathrm{C}$, cinnamic acid, and harpagoside is simple. The results indicate that it is feasible to analyze the bioactive components in S. ningpoensis by IRAE-HPLC. Therefore, the proposed method has the potential for the quality control of S. ningpoensis.

\section{Additional files}

Additional file 1: Figure $\mathbf{S 1}$. Overlaid chromatograms for calibration curves. 1 = angoroside $C_{;} 2=$ cinnamic acid; 3 = harpagoside. (DOCX $35 \mathrm{~kb}$ )

Additional file 2: Figure S2. Overlaid chromatograms for precision $(5 \mu \mathrm{g} / \mathrm{mL}, 2.5 \mu \mathrm{g} / \mathrm{mL}, 5 \mu \mathrm{g} / \mathrm{mL}$ for angoroside C, cinnamic acid and harpagoside, respectively). 1 = angoroside $\mathrm{C} ; 2=$ cinnamic acid; 3 = harpagoside. (DOCX $28 \mathrm{~kb}$ )

Additional file 3: Figure S3. Overlaid chromatograms for precision $(50 \mu \mathrm{g} / \mathrm{mL}, 25 \mu \mathrm{g} / \mathrm{mL}, 50 \mu \mathrm{g} / \mathrm{mL}$ for angoroside C, cinnamic acid and harpagoside, respectively). 1 = angoroside $C_{;} 2=$ cinnamic acid; 3 = harpagoside. (DOCX $30 \mathrm{~kb})$

Additional file 4: Figure S4. Overlaid chromatograms for precision $(200 \mu \mathrm{g} / \mathrm{mL}, 100 \mu \mathrm{g} / \mathrm{mL}, 200 \mu \mathrm{g} / \mathrm{mL}$ for angoroside C, cinnamic acid and harpagoside, respectively). 1 = angoroside $C ; 2=$ cinnamic acid; 3 = harpagoside. (DOCX $30 \mathrm{~kb}$ )

Additional file 5: Figure S5. Overlaid chromatograms for recovery. 1 = angoroside C; 2 = cinnamic acid; 3 = harpagoside. (DOCX $39 \mathrm{~kb}$ ) Additional file 6: Figure S6. Chromatogram showing the limit of quantification. $1=$ angoroside $C ; 2=$ cinnamic acid; $3=$ harpagoside (DOCX $21 \mathrm{~kb})$

\section{Abbreviations}

HBV: Hepatitis B virus; HPLC-UV: High-performance liquid chromatography with ultraviolet detection; IRAE: Infrared-assisted extraction; LOD: Limits of detection; LOQ: Limits of quantification; MAE: Microwave-assisted extraction; RSD: Relative standard deviation; S/N: Signal-to-noise; TCM: Traditional Chinese medicine; USE: Ultrasonic extraction

\section{Acknowledgments}

We are grateful to Professor Chunhui Deng for his technical assistance.

\section{Authors' contribution}

LS and TH participated in the design of this work, performed the experiments and wrote the manuscript. YD, NC, and XZ analyzed the data and supervised the work. All authors read the final manuscript and approved it for submission.

\section{Funding}

This study was financially supported by the Science and Technology Commission of Shanghai Municipality (No. 11DZ1972100, 19401900600).

Availability of data and materials

The datasets used and/or analyzed during the current study are available from the corresponding author on reasonable request.

Ethics approval and consent to participate Not applicable. 


\section{Consent for publication}

Not applicable.

\section{Competing interests}

The authors declare that they have no competing interests.

\section{Author details}

'Department of Pharmacy, Qujing Medical College, Qujing 655000, China. ${ }^{2}$ Department of Pharmacy, Eye \& ENT Hospital, Fudan University, Shanghai 200031, China.

Received: 27 June 2018 Accepted: 7 June 2019

Published online: 14 June 2019

\section{References}

1. Yu W, Ma M, Chen X, Min J, Li L, Zheng Y, et al. Traditional Chinese medicine and constitutional medicine in China, Japan and Korea: a comparative study. Am J Chin Med. 2017;45(1):1-12.

2. Huang TM, Chen NZ, Lai YH, Wang DL, Yan JC, Gu JF. Rapid determination of cinnamic acid and harpagoside in a traditional Chinese medicine of Scrophularia ningpoensis by microwave-assisted extraction followed by high performance liquid chromatography (HPLC). J Med Plants Res. 2011; 5(8):1313-20.

3. Zhu LJ, Hou YL, Shen XY, Pan XD, Zhang X, Yao XS. Monoterpene pyridine alkaloids and phenolics from Scrophularia ningpoensis and their cardioprotective effect. Fitoterapia. 2013;88:44-9.

4. Baghdikian B, Lanhers MC, Fleurentin J, Ollivier E, Maillard C, Balansard G, et al. An analytical study, anti-inflammatory and analgesic effects of Harpagophytum procumbens and Harpagophytum zeyheri. Planta Med. 1997:63(2):171-6.

5. Zhang YF, Liu L, Xu F, Shang MY, Liu GX, Cai SQ. Investigation of the In Vivo Metabolism of Sibirioside A and Angoroside C in Rats by HPLC-ESI-ITTOF-MSn. Molecules (Basel, Switzerland). 2018;23(10):14.

6. Manon L, Beatrice B, Thierry O, Jocelyne P, Fathi M, Evelyne O, et al. Antimutagenic potential of harpagoside and Harpagophytum procumbens against 1-nitropyrene. Pharmacogn Mag. 2015;11(Suppl 1):S29-36.

7. Sheu SY, Hong YW, Sun JS, Liu MH, Chen CY, Ke CJ. Radix Scrophulariae extracts (harpagoside) suppresses hypoxia-induced microglial activation and neurotoxicity. BMC Complementary Altern Med. 2015;15:324.

8. Haseeb A, Ansari MY, Haqqi TM. Harpagoside suppresses IL-6 expression in primary human osteoarthritis chondrocytes. J Orthop Res. 2017;35(2):311-20.

9. Ferrante C, Recinella L, Locatelli M, Guglielmi P, Secci D, Leporini L, et al. Protective effects induced by microwave-assisted aqueous Harpagophytum extract on rat cortex Synaptosomes challenged with amyloid beta-peptide. Phytother Res. 2017;31(8):1257-64.

10. Locatelli M, Ferrante C, Carradori S, Secci D, Leporini L, Chiavaroli A, et al. Optimization of aqueous extraction and biological activity of Harpagophytum procumbens root on ex vivo rat Colon inflammatory model. Phytother Res. 2017;31(6):937-44.

11. Korosec B, Sova M, Turk S, Krasevec N, Novak M, Lah L, et al. Antifungal activity of cinnamic acid derivatives involves inhibition of benzoate 4hydroxylase (CYP53). J Appl Microbiol. 2014;116(4):955-66.

12. Nanjundaiah SM, Annaiah HN, Dharmesh SM. Gastroprotective Effect of Ginger Rhizome (Zingiber officinale) Extract: Role of Gallic Acid and Cinnamic Acid in $\mathrm{H}(+), \mathrm{K}(+)$-ATPase/H. pylori Inhibition and Anti-Oxidative Mechanism. J Evidence-based Complementary Altern Med. 2011;2011:249487.

13. Qi G, Chen J, Shi C, Wang Y, Mi S, Shao W, et al. Cinnamic acid (CINN) induces apoptosis and proliferation in human nasopharyngeal carcinoma cells. Cell Physiol Biochem. 2016;40(3-4):589-96.

14. Wang SJ, Ruan JX, Zhao YH, Zhang ZQ. Simultaneous determination of harpagoside and cinnamic acid in rat plasma by liquid chromatography electrospray ionization mass spectrometry and its application to pharmacokinetic studies. Biomed Chromatogr. 2008;22(1):50-7.

15. Colas C, Garcia P, Popot MA, Bonnaire Y, Bouchonnet S. Liquid chromatography/electrospray ionization mass spectrometric characterization of Harpagophytum in equine urine and plasma. Rapid Commun Mass spectrom. 2006;20(22):3257-66.

16. Cao G, Wu X, Li Q, Cai H, Cai B, Zhu X. Influence of processing procedure on the quality of Radix Scrophulariae: a quantitative evaluation of the main compounds obtained by accelerated solvent extraction and highperformance liquid chromatography. J Sep Sci. 2015;38(3):390-4.
17. Li P, Zhang Y, Xiao L, Jin X, Yang K. Simultaneous determination of harpagoside and cinnamic acid in rat plasma by high-performance liquid chromatography: application to a pharmacokinetic study. Anal Bioanal Chem. 2007:389(7-8):2259-64.

18. Diuzheva A, Carradori S, Andruch V, Locatelli M. Use of innovative (micro) extraction techniques to characterise Harpagophytum procumbens root and its commercial food supplements. Phytochem Anal. 2018;29(3):233-41.

19. Locatelli M, Zengin G, Uysal A, Carradori S, De Luca E, Bellagamba G, et al. Multicomponent pattern and biological activities of seven Asphodeline taxa: potential sources of natural-functional ingredients for bioactive formulations. J Enzyme Inhib Med Chem. 2017:32(1):60-7.

20. Ehala S, Vaher M, Kaljurand M. Characterization of phenolic profiles of northern European berries by capillary electrophoresis and determination of their antioxidant activity. J Agric Food Chem. 2005;53(16):6484-90.

21. Guo FQ, Liang $Y Z, X u C J, L i X N$, Huang LF. Analyzing of the volatile chemical constituents in Artemisia capillaris herba by GC-MS and correlative chemometric resolution methods. J Pharm Biomed Anal. 2004;35(3):469-78.

22. Guo FQ, Liang YZ, Xu CJ, Huang LF. Determination of the volatile chemical constituents of Notoptergium incium by gas chromatography-mass spectrometry and iterative or non-iterative chemometrics resolution methods. J Chromatogr A. 2003;1016(1):99-110.

23. Huang LF, Li BY, Liang YZ, Guo FQ, Wang YL. Application of combined approach to analyze the constituents of essential oil from dong quai. Anal Bioanal Chem. 2004;378(2):510-7.

24. Yu J, Lei J, Yu H, Cai X, Zou G. Chemical composition and antimicrobial activity of the essential oil of Scutellaria barbata. Phytochemistry. 2004;65(7): 881-4.

25. Shishov A, Bulatov A, Locatelli M, Carradori S, Andruch V. Application of deep eutectic solvents in analytical chemistry. A review. Microchem J. 2017:135:33-8.

26. Chen Y, Duan G, Xie M, Chen B, Li Y. Infrared-assisted extraction coupled with high-performance liquid chromatography for simultaneous determination of eight active compounds in Radix Salviae miltiorrhizae. J Sep Sci. 2010;33(17-18):2888-97.

27. Li FJ, Yu YJ, Zhang HY, Liu TT, Li Y, Duan GL. Infrared-assisted non-ionic surfactant extraction as a green analytical preparatory technique for the rapid extraction and pre-concentration of picroside I and picroside II from Picrorhiza scrophulariiflora Pennell. Anal Methods. 2013;5(15):3747-53.

28. Huang TM, Chen NZ, Wang DL, Lai YH. Infrared-assisted extraction coupled with high performance liquid chromatography (HPLC) for determination of liquiritin and glycyrrhizic acid in licorice root. Anal Methods. 2014;6(15): 5986-91.

\section{Publisher's Note}

Springer Nature remains neutral with regard to jurisdictional claims in published maps and institutional affiliations.

\section{Ready to submit your research? Choose BMC and benefit from:}

- fast, convenient online submission

- thorough peer review by experienced researchers in your field

- rapid publication on acceptance

- support for research data, including large and complex data types

- gold Open Access which fosters wider collaboration and increased citations

- maximum visibility for your research: over $100 \mathrm{M}$ website views per year

At BMC, research is always in progress.

Learn more biomedcentral.com/submissions 